Original Research Paper

\title{
Analysis of Combined Natural Convection and Radiation Heat Transfer Using a Similarity Solution
}

\author{
${ }^{1}$ Mehdi Zeyghami and ${ }^{2}$ Muhammad M. Rahman \\ ${ }^{I}$ Department of Mechanical Engineering, University of South Florida, Tampa, FL, USA \\ ${ }^{2}$ Department of Mechanical Engineering, Wichita State University, Wichita, KS, USA
}

\author{
Article history \\ Received: 27-05-2015 \\ Revised: $18-09-2015$ \\ Accepted: 28-09-2015 \\ Corresponding Author: \\ Mehdi Zeyghami \\ Department of Mechanical \\ Engineering, University of \\ South Florida, Tampa, FL, \\ USA \\ E-mail: mzeyghami@mail.usf.edu
}

\begin{abstract}
A similarity solution for laminar boundary layer in a buoyancy induced flow over an isothermal, vertical wall in the presence of radiation is developed. Radiation heat transfer is taken into account by application of the Rosseland approximation in the energy conservation equation. The family of similarity solutions to the governing equations is presented. The computed results show that thermal and momentum boundary layer thickness values are increased as a result of radiation heat transfer. Furthermore for higher values of the radiation parameter, the maximum velocity inside the boundary layer increases.
\end{abstract}

Keywords: Natural Convection, Combined Radiation-Convection, Similarity Solution, Boundary Layer

\section{Introduction}

Evaluating the heat transfer interactions involving combined convection and radiation is important in many engineering applications, especially at high temperatures. Natural convection flow analysis has been the main subject of numerous researches due to its many applications in engineering and industrial processes. At high temperatures, when flowing substance is hot enough to emit and absorb radiant energy we are dealing with a combined radiation-convection heat transfer mode. Radiation effect on flow is most significant in situations where, convection heat transfer is limited; hence radiation could have a high impact on free convection instances of absorbing-emitting fluids (Cess, 1966). Energy transfer through participating media has received a lot of attention during past years. Some recent important applications are high temperature solar energy receivers, high temperature thermal storage, advanced power generation systems, radiant furnaces, molten glass containers and so on.

The receiver of a Concentrated Solar Power (CSP) plant represents an example where combined radiationconvection heat transfer could be expected. The Solar receiver of a CSP plant is the heat exchanger where the concentrated solar radiation is absorbed and converted into heat. Temperature of the solar receiver can reach more than $700{ }^{\circ} \mathrm{C}$ in a solar tower. Precise evaluation of heat losses from the structure due to convection and radiation is a complex problem. The convective and radiative heat transfer between the surface and the hot fluid adjacent to it are coupled and none of the heat transfer modes could be evaluated separately. The presence of radiative heat transfer affects the velocity and temperature profiles inside the momentum and thermal boundary layers and alters the overall heat transfer coefficient.

Although some of these applications are relatively recent, the importance of this subject has been recognized since the 1960s. Cess (1966) analyzed the interaction of thermal radiation with free convection for a gray gas in the optically thick region. He solved the problem by means of singular perturbation technique for small values of radiation parameter $\left(\mathrm{N}_{\mathrm{R}}<<1\right)$. Arpaci (1968) investigated the effect of thermal radiation on local heat flux for laminar free convection from a heated vertical plate. He approximated the radiation heat flux by an integro exponential function and selected first order polynomial to approximate the velocity and temperature profiles. Due to lengthy expressions, no results were presented for temperature and velocity profiles and only local heat flux for thick and thin gas assumptions was presented. Cheng and Özişic (1972) considered the heat transfer for both hot and cold wall by simultaneous radiation and free convection in an absorbing, emitting, isotropically scattering gray fluid by solving non-similar momentum and energy equations. The radiant part of the problem was treated by the application of a normalmode-expansion technique. Their results show that both temperature and velocity boundary layers get thicker by decreasing radiation parameter $\left(\mathrm{N}_{\mathrm{R}}\right)$. Hossain and Takhar (1996) have studied the effects of radiation on forced and free convection, boundary layer flow along a vertical flat 
plate with uniform free stream and surface temperatures. They used the Rosseland diffusion approximation for radiation, which leads to non-similarity solutions for the flow. In their findings, they declared that both dimensionless local shear stress and local heat transfer rate decline when $\mathrm{N}_{\mathrm{R}}$ increases. In another study, Hossain et al. (1999) studied the same problem for a porous vertical plate. Molla et al. (2011) investigated the effect of radiation on the natural convection flow of an optically thick viscous incompressible fluid along a vertical plate with sinusoidal temperature distribution. Siddiqa et al. (2014) investigated the effects of radiation heat transfer on the natural convection boundary layer flow over a wavy horizontal surface.

Bataller (2008a; 2008b) analyzed the effects of thermal radiation on the laminar boundary layer adjacent to a flat plate in a uniform stream of fluid (Blasius flow) and also on a moving plate in a quiescent ambient fluid (Sakiadis flow) under convective-radiative surface boundary conditions. Also, many studies on laminar and turbulent natural convection heat transfer coupled with radiation heat transfer in the presence of a radiative surface and/or participating medium in an enclosure have been published (Xamán et al., 2008; Moghadassian and Kowsary, 2014; Bouali et al., 2006). Thermal radiation effect in natural convection for a cylinder has been investigated by a number of researchers. For example, Novotny and Kelleher (1967) investigated the laminar free convection of an absorbing, emitting gas in the region of the stagnation point of a horizontal cylinder. The radiation effect on free convection along an isothermal vertical cylinder and mixed convection from a horizontal cylinder were studied by Hossain et al. (1998; Hossain and Alim, 1997; Hossain et al., 1999).

Including radiation heat transfer mode into energy equation, leads to a nonlinear partial differential equation. Almost all the previous works in this field utilized an approximate method to solve this differential or in some cases integro-differential equation. As a result, any parametric analysis of the velocity and temperature profiles was lengthy and difficult.

The main objective of the present work is to investigate the effects of radiation heat transfer on momentum and heat transfer boundary layers. The velocity and temperature distribution profiles inside the boundary layer for an optically thick, incompressible, natural buoyancy induced flow past a hot vertical wall with constant temperature are evaluated. By using Rosseland diffusion approximation and making a series of transformations on governing equations, we delivered the similarity solution for the flow field. This procedure simplifies the analysis and makes interpretation of the results easier.

\section{Governing Equations}

Consider laminar boundary layer flow over a hot flat plate with constant temperature, which is driven by the buoyancy forces depicted in Fig. 1. To simplify the problem, the following assumptions are made:

- Steady-state two-dimensional conditions in which the gravity force acts in the negative $\mathrm{x}$ direction

- General Boussinnesq assumption and taking into account the boundary layer approximation

- Gray fluid, emitting and absorbing radiation with no scattering effect

- Constant physical properties

- Viscous dissipation has been neglected in the energy, due to the small velocities associated with the free convection

The governing equations take the form of (Equations (1) to (3)):

$$
\frac{\partial u}{\partial x}+\frac{\partial v}{\partial y}=0
$$

$$
\begin{aligned}
& u \frac{\partial u}{\partial x}+v \frac{\partial u}{\partial y}=v \frac{\partial^{2} u}{\partial y^{2}}+g \beta\left(T-T_{\infty}\right) \\
& u \frac{\partial T}{\partial x}+v \frac{\partial T}{\partial y}=\frac{k}{\rho c_{p}} \frac{\partial^{2} T}{\partial y^{2}}-\frac{1}{\rho c_{p}} \frac{\partial q_{r}}{\partial y}
\end{aligned}
$$

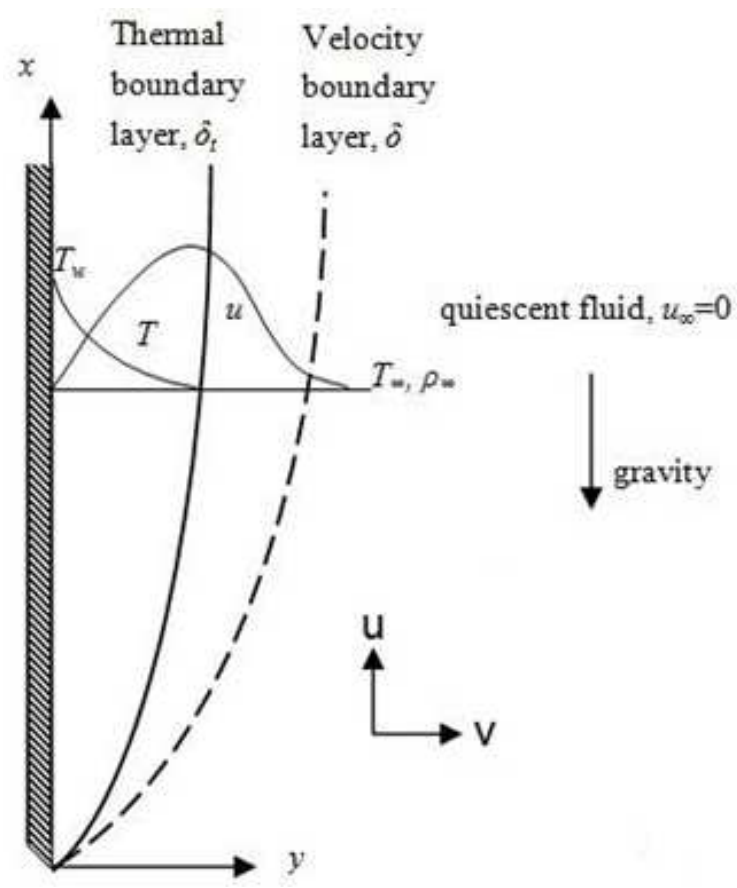

Fig. 1. Momentum and thermal boundary layer development on a hot vertical wall 
Here $\mathrm{u}$ and $\mathrm{v}$ are velocity components in $\mathrm{x}$ and $\mathrm{y}$ directions, $g$ is the acceleration due to gravity, $\beta$ is the coefficient of thermal expansion, $v$ is the kinematic viscosity, $\mathrm{T}$ is the temperature, $\mathrm{k}$ is the fluid conductivity, $\rho$ is the fluid density and $c_{p}$ is the heat capacity of the fluid.

The boundary conditions (Equation 4) for the velocity field are:

$$
\begin{array}{lll}
u=v=0 & \text { at } & y=0 \\
u=0 & \text { at } & x=0 \\
u \rightarrow 0 & \text { as } & y \rightarrow \infty
\end{array}
$$

The boundary conditions (Equation 5) for the temperature field are:

$$
\begin{array}{lll}
T=T_{w} & \text { at } & y=0 \\
T=T_{\infty} & \text { as } & y \rightarrow \infty
\end{array}
$$

Radiation heat transfer is added in the energy equation as one dimensional heat flux in y direction. Radiative heat fluxes can be approximated by the Rosseland diffusion approximation (Rosseland, 1936) for an optically dense medium, which has been used in many radiation related studies (Cess, 1966; Arpaci, 1968; Cheng and Özişik, 1972; Hossain and Takhar, 1996; Hossain et al., 1999; Molla et al., 2011; Siddiqa et al., 2014; Bataller, 2008a; 2008b) and (Hossain et al., 1998; Hossain and Alim, 1997; Hossain et al., 1999).Using the Rosseland diffusion approximation for radiation, the radiation heat flux is simplified as:

$q_{r}=-\frac{4 \sigma}{3 \alpha_{R}} \frac{\partial T^{4}}{\partial y}$

where, $\sigma$ and $\alpha_{R}$ are the Stefan-Boltzmann constant and the Roseland mean absorption coefficient, respectively. It is possible to assume the temperature profile within the flow such as that, the term $\mathrm{T}^{4}$ be expressed as a linear function of temperature. Hence, expanding $\mathrm{T}^{4}$ in a Taylor series about $\mathrm{T}_{\propto}$ and neglecting higher-order terms we get:

$$
T^{4} \cong 4 T_{\infty}^{3} T-3 T_{\infty}^{4}
$$

Using Equations (6) and (7), the energy Equation (3) becomes:

$$
u \frac{\partial T}{\partial x}+v \frac{\partial T}{\partial y}=\left(\alpha+\frac{16 \sigma T_{\infty}^{3}}{3 \rho c_{p} \alpha_{R}}\right) \frac{\partial^{2} T}{\partial y^{2}}
$$

Here $\alpha$ is the fluid thermal diffusivity. As it can be seen in equation (8), the effect of radiation is manifested in the form of enhanced thermal diffusivity.
By introducing radiation parameter as $N_{R}=\frac{k a_{R}}{4 \sigma T_{\infty}^{3}}$ Equation (8) can be rearranged as (Equation (9)):

$u \frac{\partial T}{\partial x}+v \frac{\partial T}{\partial y}=\frac{\alpha}{k_{0}} \frac{\partial^{2} T}{\partial y^{2}}$

where, $k_{0}=\frac{3 N_{R}}{3 N_{R}+4}$. The radiation parameter $\left(\mathrm{N}_{\mathrm{R}}\right)$ is the parameter that measures the importance of conduction versus radiation within the fluid (Cheng and Özişik, 1972; Sparrow and Cess, 1966). It can be seen that, at $\mathrm{k}_{0}$ $=1$, the effect of thermal radiation is not taken into account. Table 1 shows the range of $\mathrm{N}_{R}$ for carbon dioxide, ammonia vapor and water vapor. Now by defining a similarity variable it is possible to use the similarity solution to determine the velocity and temperature profiles adjacent to the plate.

\section{Similarity Variable Method}

The idea behind the similarity solution is that the velocity and temperature profiles in different $\mathrm{x}$ location in the boundary layer are geometrically similar, differing only by a stretching factor in the $\mathrm{x}$ direction (Kays et al., 2005). Similarity solutions to the laminar boundary layer equations for steady natural convection from an isothermal vertical flat plate have been known since the pioneering work of Schmidt and Beckmann (1930). Sparrow and Gregg (1958) added solutions for nonisothermal vertical plates with surface temperatures of the form $\mathrm{T}_{\mathrm{w}}-\mathrm{T}_{\propto}=A \mathrm{x}^{\mathrm{n}}$ and $\mathrm{Be}^{\mathrm{mx}}$.

In order to derive a general solution, the following forms for similarity variable $(\eta)$ (Equation (10)), stream function $(\psi)$ (Equation (11)) and dimensionless temperature $(\theta)$ (Equation (12)), are considered:

$$
\begin{aligned}
& \eta=\frac{y}{x}\left(\frac{1}{4} G r_{x}\right)^{1 / 4} \\
& \psi(x, \eta)=4 v F(\eta)\left(\frac{1}{4} G r_{x}\right)^{1 / 4} \\
& \theta=\frac{T-T_{\infty}}{T_{w}-T_{\infty}}=\theta(\eta)
\end{aligned}
$$

where, $F$ is the velocity function and $\mathrm{Gr}_{\mathrm{x}}$ is the local Grashof number defined as (equation (13)):

$$
G r_{x}=\frac{g \beta\left(T_{w}-T_{\infty}\right) x^{3}}{v^{2}}
$$

The velocity components in the $\mathrm{x}$ (Equation (14)) and $y$ (Equation (15)) directions can be expressed in terms of similarity variables by the following equations: 


$$
\begin{aligned}
& u=\frac{\partial \psi}{\partial y}=\frac{2 v}{x} G r_{x}^{1 / 2} F^{\prime}(\eta) \\
& v=-\frac{\partial \psi}{\partial x}=\frac{v}{x}\left(\frac{G r_{x}}{4}\right)^{1 / 4}\left[\eta F^{\prime}(\eta)-3 F(\eta)\right]
\end{aligned}
$$

Here the prime denotes differentiation with respect to $\eta$. The stream function has been introduced in a way that automatically satisfies the continuity equation. The momentum (Equation (2)) and energy (Equation (9)) equations, using the above transformations reduce to (Equation (16) and Equation (17)):

$$
\begin{aligned}
& F^{\prime \prime \prime}+\theta+3 F F^{\prime \prime}-2\left(F^{\prime}\right)^{2}=0 \\
& \theta^{\prime \prime}+3 k_{0} P r F \theta^{\prime}=0
\end{aligned}
$$

The boundary conditions transform to (equation (18) and equation (19)):

$$
\begin{aligned}
& F(\eta)=F^{\prime}(\eta)=0, \quad \theta(\eta)=1 \text { at } \eta=0 \\
& F^{\prime}(\eta)=0, \quad \theta(\eta)=0 \quad \text { as } \eta \rightarrow \infty
\end{aligned}
$$

Without considering the radiation, the Prandtl number appears explicitly in Equation (17) and the solutions are expected to be of the form $\mathrm{F}=\mathrm{F}(\eta, \operatorname{Pr})$ and $\theta=\theta(\eta, \operatorname{Pr})$. However, when radiation effects are considered, another parameter must be taken into account, which is the radiation parameter. It is possible to present the effect of radiation heat transfer by introducing the modified Prandtl number as $\operatorname{Pr}^{*}=\mathrm{k}_{0} \operatorname{Pr}$. Solutions for different Prandtl numbers are available in many references (Sparrow and Cess, 1966; Schuh, 1948; Ostrach, 1953; Webb, 1990) and it is possible to exploit desirable results for modified Prandtl number.

\section{Numerical Solution and Results}

In order to visualize the effect of radiation heat transfer, in Table 2, the values of $\operatorname{Pr}^{*} / \operatorname{Pr}$ for different values of $\mathrm{N}_{R}$ are presented. By increasing the value of $\mathrm{N}_{\mathrm{R}}$ the ratio of the convection heat transfer rate to radiation heat transfer rate increases. For small values of radiation parameter, radiation is the dominating heat transfer mode. By increasing the value of $\mathrm{N}_{\mathrm{R}}$, ratio of the convection heat transfer to radiation heat transfer increases. As it is shown in Table 2 the values of modified Prandtl number to conventional Prandtl number is always less than one $\left(\mathrm{k}_{0}<1\right)$. With the increasing of radiation parameter this ratio converges to one and for $\mathrm{N}_{R}$ more than 50, this ratio remains close to one. It is obvious as $\mathrm{N}_{R}$ increases, the value of $\mathrm{k}_{0}$ becomes closer to unity and the effect of radiation becomes less considerable on the flow.

Equations (16) and (17) are coupled nonlinear ordinary differential equations. Therefore any changes in parameters $\operatorname{Pr}^{*}$ will affect both fluid velocity and its temperature distribution in the boundary layer.
Table 1. Different gases and their $\mathrm{N}_{\mathrm{R}}$ values (Ali et al., 1984)

\begin{tabular}{llll}
\hline & $\mathrm{T}\left[{ }^{\circ} \mathrm{F}\right]$ & $\mathrm{Pr}$ & $\mathrm{NR}$ \\
\hline Carbon dioxide & $100-650$ & $0.76-0.60$ & $10-30$ \\
Ammonia vapor & $120-400$ & $0.88-0.84$ & $30-200$ \\
Water vapor & $220-900$ & 1 & $30-200$ \\
\hline
\end{tabular}

Table 2. Different gases and their $\mathrm{N}_{\mathrm{R}}$ values (Ali et al., 1984)

\begin{tabular}{ll}
\hline $\mathrm{NR}$ & $\operatorname{Pr}^{*} / \operatorname{Pr}$ \\
\hline 0.01 & 0.0074 \\
0.1 & 0.0700 \\
0.5 & 0.2720 \\
1 & 0.4290 \\
5 & 0.7900 \\
15 & 0.9180 \\
25 & 0.9380 \\
50 & 0.9740 \\
100 & 0.9870 \\
1000 & 0.9990 \\
\hline
\end{tabular}

Equation (17) is a second order ordinary differential equation and can be broken down into two first order ordinary differential equations (equation (20) and equation (21)):

$\theta^{\prime}=A$

$A^{\prime}=-3 \operatorname{Pr}^{*} F \theta^{\prime}=g(F, A)$

Equation (16) is a third order ordinary differential equation and can be broken down into three first order ordinary differential equations (equations (22) to (24)):

$$
\begin{aligned}
& F^{\prime}=B \\
& B^{\prime}=C \\
& C^{\prime}=2 B^{2}-3 F C-\theta=f(B, F, C, \theta)
\end{aligned}
$$

By use of forth order Runge-Kutta algorithm along with shooting procedure these equations are solved simultaneously. There are three boundary conditions at $\eta$ $=0$ which are: $F(0)=F^{\prime}(0)=0$ and $\theta(0)=1$ and two boundary conditions at $\eta \rightarrow \propto$ which are: $F^{\prime}(\infty)=\theta(\infty)=0$. We guess the values of $F^{\prime \prime}(0)$ and $\theta^{\prime}(0)$ and compare the calculated values at the other end of the domain $(\eta \rightarrow \infty)$ with the existing boundary conditions. This procedure continues until the desirable convergence between calculated values and boundary values are reached. The problem was solved with $\Delta_{\eta}=0.01$ and a complete determination of the solution has been carried out.

There are very limited experimental studies available on effect of combined radiation-convection heat transfer inside boundary layer in literature. Webb (1990) conducted an experimental and analytical study to explore the interaction between laminar free convection and radiative heat transfer from an isolated vertical plate with isoflux heating. Experiments were formulated and carried out in air environment at moderate pressure, therefore negligible volumetric radiation-absorption was 
considered inside the boundary layer. A similar study by Sabareesh et al. (2010) was carried out for a hot plate with isoflux and isothermal boundary conditions. In an experimental study the effects of gas-gas radiation on thermal fields in a gas flowing by free convection around a finite isothermal plate was investigated by Lacona and Taine (2001). Holographic interferometry and laser beam deflection techniques were used to show the radiation heat transfer effects on boundary layer for both absorbing $\left(\mathrm{CO}_{2}\right)$ and non-absorbing $\left(\mathrm{N}_{2}\right)$ mediums. Although temperature distribution perpendicular to the plate and local heat transfer coefficient along the plate were presented for non-absorbing medium, for absorbing-emitting medium only the heat transfer coefficient is reported.

To compare the modified Prandtl number with experimental results following equations are used for calculating the local heat transfer coefficient along the plate (equation (25)):

$h_{x}=\frac{k}{x}\left(-\frac{\theta^{\prime}(0)}{\sqrt{2}}\right) G r_{x}^{1 / 4}$

Here $\mathrm{Gr}_{\mathrm{x}}$ is the local Grashof number and $\mathrm{k}$ is thermal conductivity of the medium. The thermo-physical properties of the medium $\left(\mathrm{CO}_{2}\right)$ is calculated at film temperature (equation (26)):
$T_{f}=\frac{T_{w}+T_{\infty}}{2}$

where, $T_{w}$ is the wall temperature and $T_{\infty}$ is considered as the cavity temperature equal to temperature of undisturbed fluid. Figure 2-4 show the calculated heat transfer coefficient and experimental data from (Lacona and Taine, 2001). Three different conditions for $\mathrm{T}_{\mathrm{w}}$ and $\mathrm{T}_{\propto}$ were analyzed.

As mentioned by Lacona and Taine (2001; Gebhart et al., 1988), the gas has been preheated by convection along horizontal edge of the plate and radiation from cavity walls; also boundary layer thickness does not change considerably in the range of $10-40 \mathrm{~mm}$ and approximately corresponds to the difference between theoretical and experimental values. Also the optically thick approximation is not completely valid at the leading edge of the plate, which makes the analytical results lower the experimental data.

The average difference between experimental and analytical results is $21.5 \%$.

To examine the outcome of modified Prandtl method with other analytical methods, comparisons have been made with results presented by Hossain et al. (1999) and Cheng and Özişic (1972).

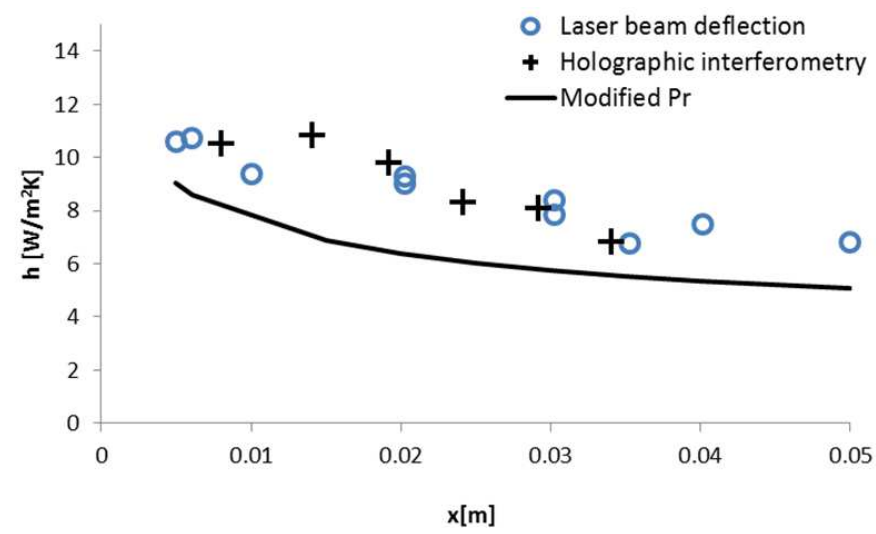

Fig. 2. Local heat transfer coefficient along the plate, $T_{w}=523 \mathrm{~K}, \mathrm{~T}_{\infty}=473 \mathrm{~K}, \mathrm{~N}_{\mathrm{R}}=22, \operatorname{Pr}=0.74$

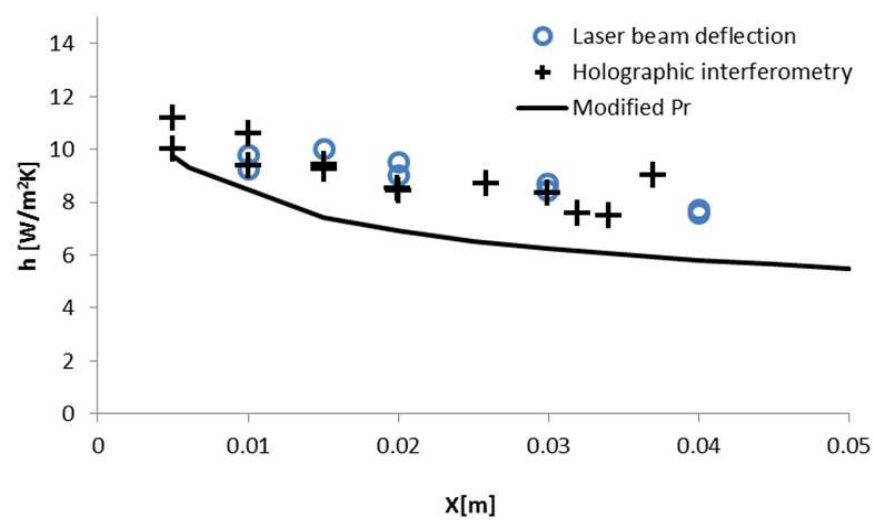

Fig. 3. Local heat transfer coefficient along the plate, $T_{w}=603 \mathrm{~K}, T_{w}=543 \mathrm{~K}, N_{R}=27, \operatorname{Pr}=0.73$ 
Figure 5 and 6 show the effect of radiation on velocity and temperature distribution for a fluid with $\operatorname{Pr}=1$ and $\mathrm{N}_{\mathrm{R}}=1.0$. The results using modified Prandtl number are very close to the results presented by Hossain et al. (1999).

Figure 7 and 8 show the effect of radiation on boundary layer velocity and temperature distributions for a fluid with $\operatorname{Pr}=0.733$ and $N_{R}=1.0$. The results for temperature distribution using modified Prandtl number are very close to the results presented by Cheng and Özişic (1972), but velocity profile which is presented by Cheng and Özişic (1972) is not for optically thick gas and therefore some mismatch is expected.

As it is depicted in Figures 5 through 8, the maximum flow velocity inside the boundary layer is up to $26.3 \%$ higher for $\operatorname{Pr}=1.0$ and up to $24.4 \%$ higher for $\operatorname{Pr}=0.733$, when radiation heat transfer is considered compared to no radiation case. For fluid with larger value of Pr number, the effect of radiation heat transfer inside the momentum boundary layer is more prominent with respect to the fluid with a lower Pr number.

Furthermore, it is shown that the temperature distribution profile inside the boundary layer becomes more uniform due to presence of radiation heat transfer compered to no radiation case. In addition both momentum and thermal boundary layers get thicker when radiation heat transfer is present. Further details are presented in Fig. 9 and 10 and Table 3.

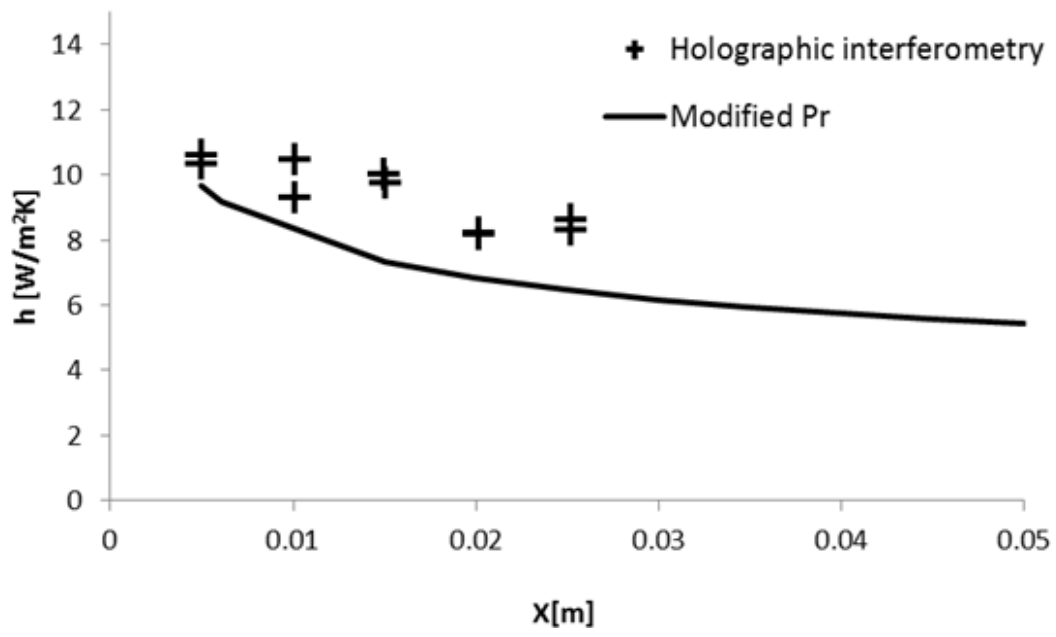

Fig. 4. Local heat transfer coefficient along the plate, $T_{w}=643 \mathrm{~K}, T_{w}=583 \mathrm{~K}, \mathrm{~N}_{\mathrm{R}}=29, \operatorname{Pr}=0.72$

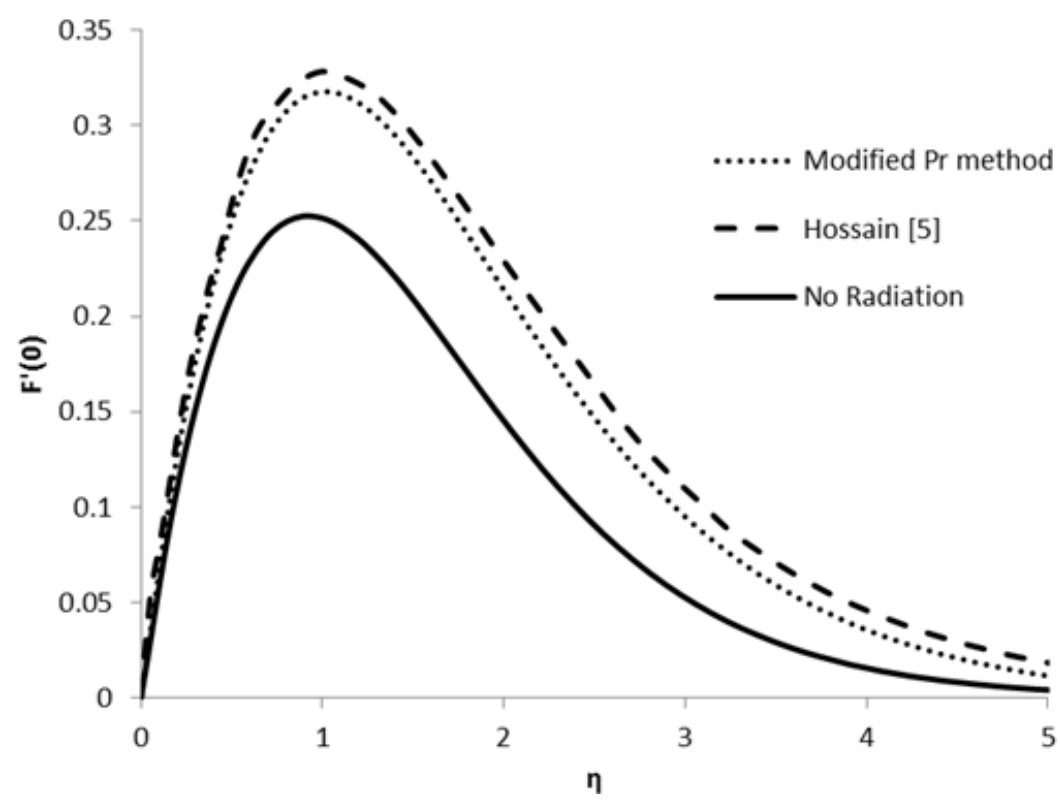

Fig. 5. Velocity distribution as a function of $\eta, N_{R}=1.0, \operatorname{Pr}=1.0$ 


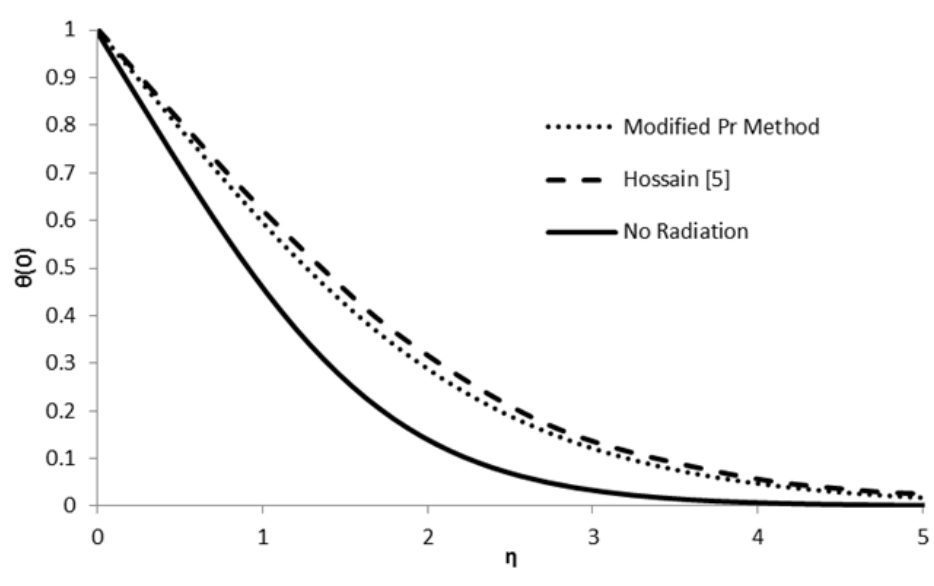

Fig. 6. Temperature distribution as a function of $\eta, N_{R}=1.0, \operatorname{Pr}=1.0$

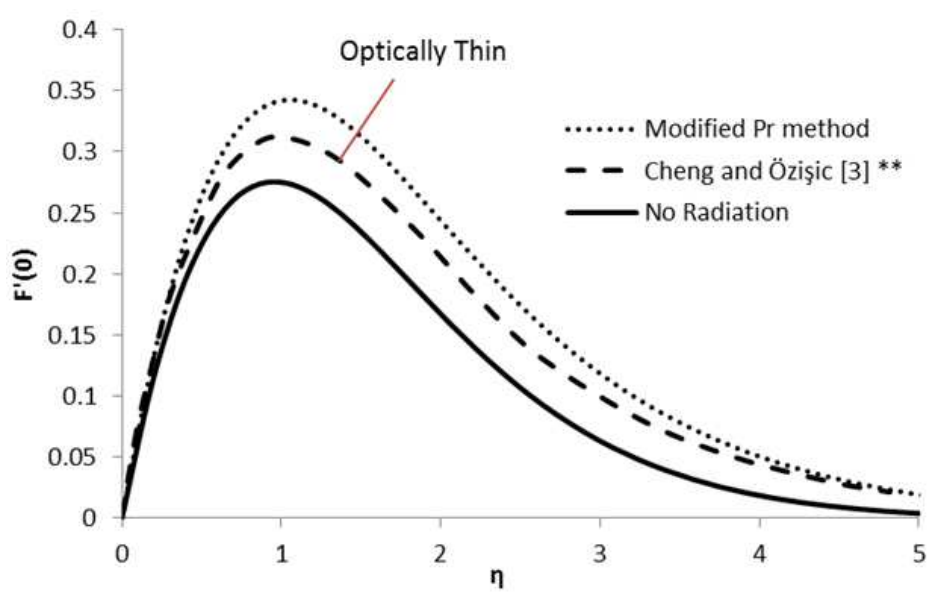

Fig. 7. Velocity distribution as a function of $\eta, N_{R}=1.0, \operatorname{Pr}=0.733$

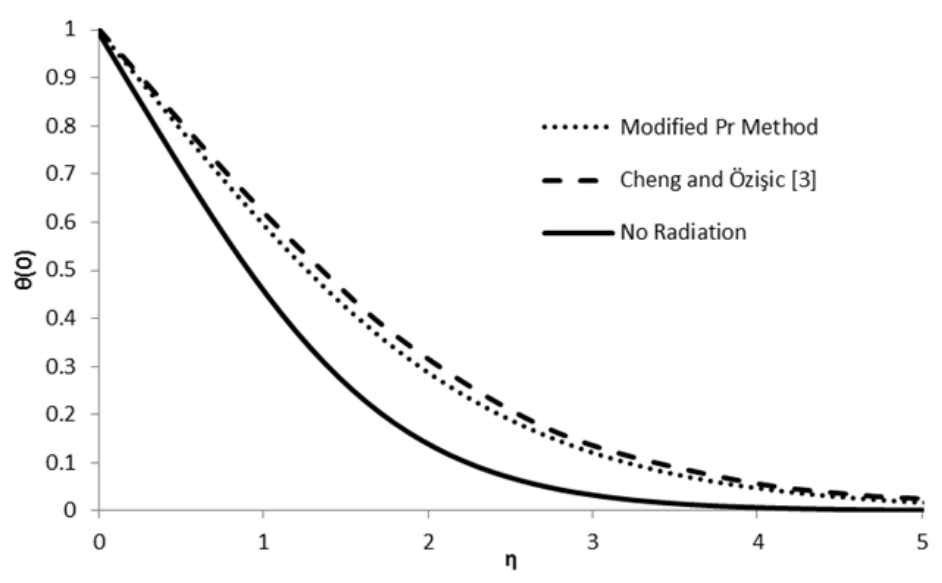

Fig. 8. Temperature distribution as a function of $\eta, N_{R}=1.0, \operatorname{Pr}=0.733$

The results for distribution of $F^{\prime}(\eta) \infty u$ and $\theta(\eta)$ in a range of $\mathrm{N}_{\mathrm{R}}$ numbers between 0.5 and 10 are shown in Fig. 9 and 10, which are in the acceptable range in accordance with data presented in (Gebhart et al., 1988) for no radiation condition and for different Prandtl numbers.
Numerical value of $\mathrm{k}_{0}$ is always between zero and one, therefore the value of $\operatorname{Pr}^{*}$ will always be less than the value of Pr. Table 3 presents the enhancement of momentum boundary layer thickness and maximum velocity inside the boundary layer for different $\mathrm{N}_{R}$ numbers compared to no radiation condition. 


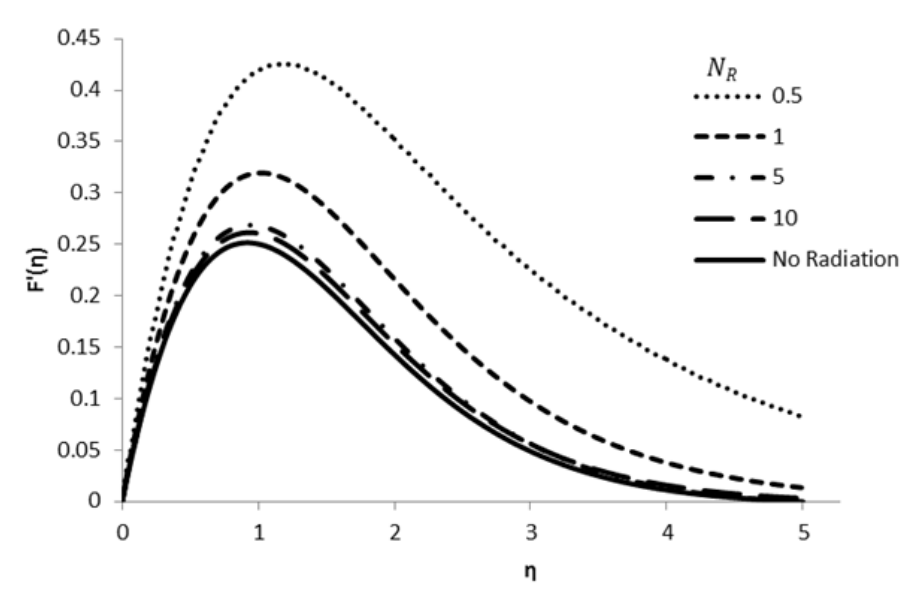

Fig. 9. Boundary region velocity distribution as a function of $\eta$ for different $N_{R}$ at $\operatorname{Pr}=1$

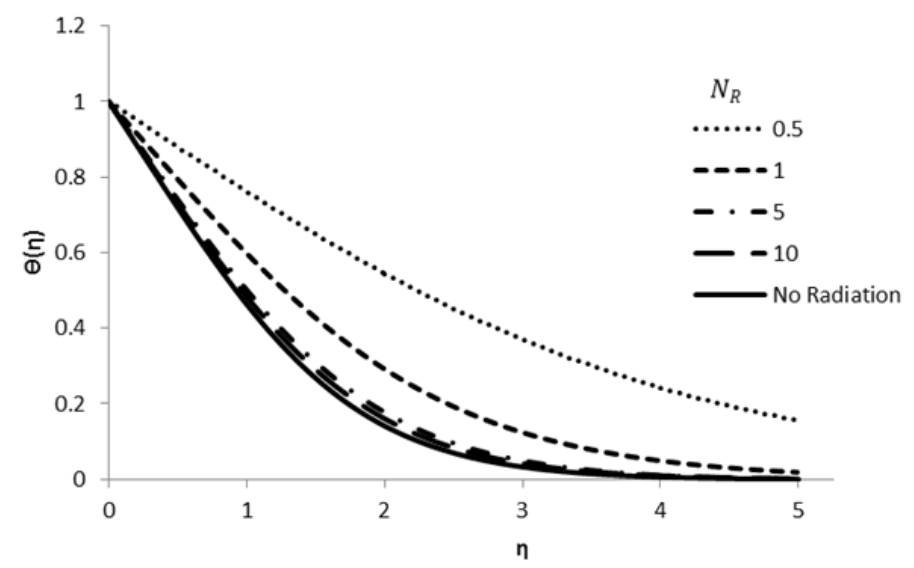

Fig. 10. Boundary region temperature distribution as a function of $\eta$ for different $N_{R}$ at $\operatorname{Pr}=1$

Table 3. Enhancement of momentum boundary layer thickness and maximum velocity in boundary layer for different $\mathrm{NR}$, at $\mathrm{Pr}=1$

\begin{tabular}{lll}
\hline NR & Enhancement of boundary layer thickness & Enhancement of max. velocity inside the boundary layer \\
\hline (no radiation) & 1.00 & 1.00 \\
10 & 1.01 & 1.03 \\
5 & 1.04 & 1.07 \\
1 & 1.20 & 1.26 \\
0.5 & 1.46 & 1.41 \\
\hline
\end{tabular}

As it is presented in the Table 3 by reduction of $\operatorname{Pr}^{*}$ (lower radiation parameter), thickness of momentum boundary layer increases and also the maximum value of velocity inside the boundary layer increases. Similar conclusions are reported by Luo et al. (2014). For $\mathrm{N}_{R}$ value equal to 0.5 the momentum boundary thickness is enhanced by $46 \%$ and maximum velocity inside the boundary layer increases by $41 \%$ at $\operatorname{Pr}=1$.

\section{Conclusion}

In this study, the effect of radiation on laminar natural convection flow adjacent to a vertical isothermal wall has been investigated. Unlike other researches done in this area, the introduced analytical procedure provides an opportunity to incorporate the effects of radiation fast and with fewer amounts of calculations. Radiation heat transfer effect is taken into account by Rosseland approximation and considering the radiation parameter in governing energy balance equation. This parameter is a tool to determine the importance of radiation heat transfer in fluid flow inside the boundary layer. It is illustrated that as the value of radiation parameter increases, a diminution in the thermal radiation effect appears.

By manipulating the energy equation and using the similarity solution method, two ordinary differential equations have been established to determine the 
velocity and temperature profiles inside the boundary layer. The only difference of these equations with conventional natural convection equations is a new parameter $\left(\mathrm{k}_{0}\right)$ which is multiplied to Prandtl number and resulting in a modified Prandtl number. Because the value of $\mathrm{k}_{0}$ is always less than one, the value of modified Prandtl number is always less than the Prandtl number. Hence, the presence of radiative heat transfer results in increasing the thickness of the momentum boundary layer and also increasing the maximum fluid velocity inside the boundary layer.

Effect of radiation heat transfer on the velocity and temperature profiles in the boundary layer for most of gases is not considerable for $\mathrm{N}_{R} \geq 10$ and can be ignored for $N_{R}$ higher than 50. But for lower values of $N_{R}$, neglecting radiation heat transfer will result in significant errors in heat transfer coefficient and skin friction.

\section{Author's Contributions}

Mehdi Zeyghami: As the first and corresponding author discovered the new method of solving the problem, executed the numerical analysis and extracted the results and, contributed to the writing of the manuscript.

Muhammad M. Rahman: As the second author of the paper designed the research plan and organized the study and contributed to the writing of the manuscript

\section{Ethics}

We maintained highest possible ethical standards Integrity regarding data gathering and analysis

\section{References}

Ali, M.M., T. Chen and B. Armaly, 1984. Natural convection-radiation interaction in boundary-layer flow over horizontal surfaces. AIAA J., 22: 1797-1803. DOI: $10.2514 / 3.8854$

Arpaci, V.S., 1968. Effect of thermal radiation on the laminar free convection from a heated vertical plate. Int. J. Heat Mass Trans., 11: 871-81. DOI: $10.1016 / 0017-9310(68) 90130-0$

Bataller, C.R., 2008a. Radiation effects in the Blasius flow. Appl. Math. Comput., 198: 333-338. DOI: 10.1016/j.amc.2007.08.037

Bataller, R.C., 2008b. Radiation effects for the Blasius and Sakiadis flows with a convective surface boundary condition. Appl. Math. Comput., 206: 832-840. DOI: 10.1016/j.amc.2008.10.001

Bouali, H., A. Mezrhab, H. Amaoui and M. Bouzidi, 2006. Radiation-natural convection heat transfer in an inclined rectangular enclosure. Int. J. Therm. Sci., 45: 553-566. DOI: 10.1016/j.ijthermalsci.2005.10.001

Cess, R.D., 1966. The interaction of thermal radiation with free convection heat transfer. Int. J. Heat Mass Trans., 9: 1269-77.
Cheng, E.H. and M.N. Özişik, 1972. Radiation with free convection in an absorbing, emitting and scattering medium. Int. J Heat Mass Trans., 15: 1243-1252. DOI: 10.1016/0017-9310(72)90188-3

Gebhart, B., Y. Jaluria, R.L. Mahajan and B. Sammakia, 1988. Buoyancy-Induced Flows and Transport. Hemisphere Publishing Corporation, Washington, ISBN-10: 0891164022, pp: 1001.

Hossain, M., M. Alim and D. Rees, 1999. The effect of radiation on free convection from a porous vertical plate. Int. J. Heat Mass, 42: 181-91. DOI: 10.1016/S0017-9310(98)00097-0

Hossain, M.A. and H.S. Takhar, 1996. Radiation effect on mixed convection along a vertical plate with uniform surface temperature. Heat Mass Trans., 31: 243-248. DOI: 10.1007/BF02328616

Hossain, M.A. and M.A. Alim, 1997. Natural convection-radiation interaction on boundary layer flow along a thin vertical cylinder. Heat Mass Trans., 32: 515-520.

Hossain, M.A., M. Kutubuddin and I. Pop, 1999. Radiation-conduction interaction on mixed convection from a horizontal circular cylinder. Heat Mass Trans., 35: 307-314.

Hossain, M.A., M.A. Alim and D.A.S. Rees, 1998. Effect of thermal radiation on natural convection over cylinders of elliptic cross section. Acta Mech., 129: 177-86.

Kays, W.M., M. Crawford and B. Weigand, 2005. Convective Heat and Mass Transfer. 4th Edn., McGrawHill.

Lacona, E. and J. Taine, 2001. Holographic interferometry applied to coupled free convection and radiative transfer in a cavity containing a vertical plate between 290 and 650 K. Int. J. Heat Mass Trans., 44: 3755-64. DOI: 10.1016/S0017-9310(01)00027-8

Luo, K., H.L. Yi and H.P. Tan, 2014. Radiation effects on bifurcation and dual solutions in transient natural convection in a horizontal annulus. AIP Adv., 4: 057123. DOI: $10.1063 / 1.4879809$

Moghadassian, B. and F. Kowsary, 2014. Inverse boundary design problem of natural convection-radiation in a square enclosure. Int. J. Therm. Sci., 75: 116-126. DOI: 10.1016/j.ijthermalsci.2013.07.023

Molla, M.M., S.C. Saha and M.A. Hossain, 2011. Radiation effect on free convection laminar flow along a vertical flat plate with streamwise sinusoidal surface temperature. Math. Comput. Model, 53: 1310-9. DOI: 10.1016/j.mcm.2010.12.017

Novotny, J.L. and M.D. Kelleher, 1967. Free-convection stagnation flow of an absorbing-emitting gas. Int. J. Heat Mass Trans., 10: 1171-1178. DOI: 10.1016/0017-9310(67)90082-8

Ostrach, S., 1953. NASA report 1111. Washington. 
Rosseland, S., 1936. Theoretical Astrophysics. At the Clarendon Press, Oxford, pp: 335.

Sabareesh, R.K., S. Prasanna and S.P. Venkateshan, 2010. Investigation on multimode heat transfer from a heated vertical plate. J. Heat Transf., 132: 32501-32508.

Schmidt, E. and W. Beckmann, 1930. Das Temperatur-und Geschwindigkeitsfeld vor einer Wärme abgebenden senkrechten Platte bei natürlicher Konvektion. Tech. Mech. Und Thermodyn, 1: 391-406.

DOI: $10.1007 / \mathrm{BF} 02640222$

Schuh, H., 1948. Boundary layers of temperature in boundary layers.

Siddiqa, S., M.A. Hossain and S.C. Saha, 2014. The effect of thermal radiation on the natural convection boundary layer flow over a wavy horizontal surface. Int. J. Therm. Sci., 84: 143-50.

DOI: $10.1016 /$ j.ijthermalsci.2014.05.006

Sparrow, E. and R. Cess, 1966. Radiation Heat Transfer. 1st Edn., Wadsworth, Belmount, California.

Sparrow, F.M. and J.L. Gregg, 1958. Similar solutions for laminar free convection from a non-isothermal vertical plate. Tran. Am. Sec. Mech. Eng., 80: 379-386.

Webb, B., 1990. Interaction of radiation and free convection on a heated vertical plate-Experiment and analysis. J. Thermophys Heat Trans., 4: 117-120.

Xamán, J., J. Arce, G. Álvarez and Y. Chávez, 2008. Laminar and turbulent natural convection combined with surface thermal radiation in a square cavity with a glass wall. Int. J. Therm. Sci., 47: 1630-1638. DOI: $10.1016 /$ j.ijthermalsci.2008.01.012

\section{Nomenclature}

$\mathrm{c}$

F

$\mathrm{g}$

$\mathrm{Gr}_{\mathrm{x}}$

$\mathrm{h}_{\mathrm{x}}$

$\mathrm{k}$

$\mathrm{N}_{\mathrm{R}}$

$\mathrm{Pr}_{\mathrm{r}}$

$\operatorname{Pr}^{*}$

qr

$\mathrm{T}$

$\mathrm{T}_{\mathrm{W}}$

$\mathrm{T}_{\propto}$

$\mathrm{u}$

$\mathrm{v}$

$v$

$V$

$\mathrm{x}$

$\mathrm{y}$

\section{Greek Symbols}

$\alpha$

Fluid thermal diffusivity $\left[\mathrm{m}^{2} / \mathrm{s}\right]$

$\alpha_{R} \quad$ Rosseland mean absorption coefficient

$\beta \quad$ Coefficient of thermal expansion $[1 / \mathrm{K}]$

$\rho \quad$ Density $\left[\mathrm{kg} / \mathrm{m}^{2}\right]$

$\sigma$

$\eta$

$\psi$

$\theta$

$\delta_{\mathrm{t}}$

$\delta$
Specific heat at constant pressure $[\mathrm{J} / \mathrm{kgK}]$

Dimensionless stream function

Local Grashof number

Local heat transfer coefficient $\left[\mathrm{W} / \mathrm{m}^{2} \mathrm{~K}\right]$

Thermal conductivity [W/mK]

Modified Prandtl number

Temperature $[\mathrm{K}]$

Wall temperature [K]

Ambient fluid temperature $[\mathrm{K}]$

elocity component in $\mathrm{x}$-direction $[\mathrm{m} / \mathrm{s}]$

Kinematic viscosity $[\mathrm{kg} / \mathrm{ms}]$

Coordinate along wall surface $[\mathrm{m}]$

Coordinate normal to the wall surface[m]
Similarity variable

Stream function

Dimensionless temperature function

Thermal boundary layer thickness [m]

Velocity boundary layer thickness [m] 